\title{
Probing the Time Structure of the Quark-Gluon Plasma with Top Quarks
}

\author{
Liliana Apolinário, ${ }^{1,2}$ José Guilherme Milhano, ${ }^{1,2,3}$ Gavin P. Salam, ${ }^{3, *}$ and Carlos A. Salgado ${ }^{4}$ \\ ${ }^{1}$ LIP, Avenida Prof. Gama Pinto, 2, P-1649-003 Lisboa, Portugal \\ ${ }^{2}$ Instituto Superior Técnico (IST), Universidade de Lisboa, Avenida Rovisco Pais 1, 1049-001 Lisbon, Portugal \\ ${ }^{3}$ CERN, Theoretical Physics Department, CH-1211 Geneva 23, Switzerland \\ ${ }^{4}$ Instituto Galego de Física de Altas Enerxías (IGFAE), Universidade de Santiago de Compostela, \\ E-15782 Santiago de Compostela, Galicia, Spain
}

(Received 30 November 2017; revised manuscript received 27 March 2018; published 8 June 2018)

\begin{abstract}
The tiny droplets of quark gluon plasma (QGP) created in high-energy nuclear collisions experience fast expansion and cooling with a lifetime of a few $\mathrm{fm} / c$. Despite the information provided by probes such as jet quenching and quarkonium suppression, and the excellent description by hydrodynamical models, direct access to the time evolution of the system remains elusive. We point out that the study of hadronically decaying $W$ bosons, notably in events with a top-antitop quark pair, can provide key novel insight into the time structure of the QGP. This is because of a unique feature, namely a time delay between the moment of the collision and that when the $W$-boson decay products start interacting with the medium. Furthermore, the length of the time delay can be constrained by selecting specific reconstructed top-quark momenta. We carry out a Monte Carlo feasibility study and find that the LHC has the potential to bring first limited information on the time structure of the QGP. Substantially increased LHC heavy-ion luminosities or future higher-energy colliders would open opportunities for more extensive studies.
\end{abstract}

DOI: 10.1103/PhysRevLett.120.232301

The quark-gluon plasma (QGP), a state that characterized the first microseconds of the Universe, is regularly produced and studied in ultrarelativistic heavy-ion collisions at both RHIC and the LHC. A range of complementary probes is used to study the QGP. These include properties that can be ascribed to hydrodynamic flow patterns, suppression of heavy-quark bound states, hadrochemistry of the final state, and modifications of the fragmentation of energetic partons that traverse the medium (see, e.g., Ref. [1]). A property common to all these probes is that they are sensitive to the properties of the QGP integrated over its lifetime.

Hydrodynamic simulation codes [2] predict a strong time dependence of the QGP's properties associated with its expansion and cooldown, which last about $10 \mathrm{fm} / c$ at the LHC. It would be invaluable to develop a way of probing this time dependence. The recent discovery (see, e.g., Refs. [3,4] and references therein) that high-multiplicity proton-proton $(p p)$ and proton-nucleus $(p A)$ collisions show signatures suggestive of collective effects, in systems with significantly smaller lifetimes than typical $\mathrm{PbPb}$ or

Published by the American Physical Society under the terms of the Creative Commons Attribution 4.0 International license. Further distribution of this work must maintain attribution to the author(s) and the published article's title, journal citation, and DOI. Funded by SCOAP .
AuAu collisions, is an additional motivation for devising a way of probing the time structure of the QCD medium.

One powerful probe of the QGP is "jet quenching," i.e., the study of modifications of jets that pass through the QGP (see, e.g., Ref. [5]). In all hard processes used so far for this purpose, dijet, $\gamma+$ jet or $Z+$ jet production, the jets are produced simultaneously with the collision of the ions.

In this Letter, we point out that top-antitop $(t \bar{t})$ production offers a unique novel opportunity to study the quarkgluon plasma, in particular its time structure. This is because, at variance with all other jet measurements considered so far in the literature, the jets that come from the decay products of the $W$ boson start interacting with the medium only at later times, due to a series of time delays [6]. At rest, top quarks decay with a lifetime of about $\tau_{\text {top }} \simeq$ $0.15 \mathrm{fm} / c$ and the $W$ that is produced in the top-quark decay has a lifetime of about $\tau_{W} \simeq 0.09 \mathrm{fm} / c$. When the $W$ boson decays hadronically, the resulting color-singlet quark-antiquark $(q \bar{q})$ pair is not immediately resolved by the medium [9]. Only after the $q$ and $\bar{q}$ have propagated and separated a certain distance do they start interacting independently with the medium. We call this delay a decoherence time, $\tau_{d}$. Thus, the jets that are produced in the $t \rightarrow b+W \rightarrow q \bar{q}$ decay chain do not see the full QGP, but only the part of the QGP that remains after the sum of decay and decoherence times. That sum of times is correlated to the momentum of the top quark, a feature that may be exploited given a sufficient number of events. 
To carry out a first investigation of the potential of using top quarks for probing the time structure of the QGP, we proceed as follows. We take the average total delay time before the $W$ decay products start interacting with the medium to be

$$
\left\langle\tau_{\text {tot }}\right\rangle=\gamma_{t, \text { top }} \tau_{\text {top }}+\gamma_{t, W} \tau_{W}+\tau_{d},
$$

For the decay times, we use a transverse boost factor, $\gamma_{t, X}=\left(p_{t, X}^{2} / m_{X}^{2}+1\right)^{1 / 2}$, defined in terms of the mass $m_{X}$, and transverse momentum $p_{t, X}$ of particle $X$. The transverse component is the natural choice, because the frame in which the top quark has no longitudinal momentum is also the one in which it is most natural to describe its interaction with the QGP, which is approximately longitudinally invariant. We take the average decoherence time to be [9]

$$
\tau_{d}=\left(\frac{12}{\hat{q} \theta_{q \bar{q}}^{2}}\right)^{1 / 3},
$$

in natural units, $\hbar=c=1$, and with $\theta_{q \bar{q}}$ the opening angle between the two decay products of the $W$, again in a longitudinal frame where the $z$ component of $W$ momentum is zero. The quantity $\hat{q}$ is the transport coefficient of the medium (squared transverse momentum broadening per unit length, see, e.g., Ref. [5]). While in practice it is expected to be a function of time, for our proof of principle illustration here, we take it to be constant, $\hat{q}=4 \mathrm{GeV}^{2} / \mathrm{fm}$ (conservatively taken larger than found in Refs. $[10,11]$ ). To get an event-by-event estimate of the interaction start time, we will associate each component with a randomly distributed exponential distribution. With these choices, for inclusive top-quark production at the LHC with center-ofmass energy (per nucleon pair) $\sqrt{s_{N N}}=5.5 \mathrm{TeV}$, the average times are, $\left\langle\gamma_{t, \text { top }} \tau_{\text {top }}\right\rangle \simeq 0.18,\left\langle\gamma_{t, W} \tau_{W}\right\rangle \simeq 0.14$, and $\left\langle\tau_{d}\right\rangle \simeq 0.34 \mathrm{fm} / c$, with dispersions that are comparable. The $1 / 3$ power in Eq. (2), means that $\left\langle\tau_{d}\right\rangle$ is only weakly dependent on the value of $\hat{q}$.

To probe jet quenching and its time dependence in $t \bar{t}$ production, we here suggest measuring the invariant mass $m_{j j}$ of the dijet system that is produced from hadronic $W$ decays. In $p p$ events, $m_{j j}$ is closely related to the $W$ mass, modulo final-state-radiation (FSR) effects. The difference in reconstructed $m_{j j}$ in central ion-ion $(A A)$ collisions as compared to $p p$ will be our measure of jet quenching.

To evaluate the potential of such a study we examine semimuonic $t \bar{t}$ events, i.e., where one top decays to $b W(W \rightarrow \mu \nu)$, while the other decays hadronically to $b W(W \rightarrow j j)$. In $p A$ collisions it has been demonstrated that it is possible to tag this class of events with essentially no background [12] as long as two $b$ tags are required, and so we only consider signal events.

For a quantitative analysis, we use events from the "hvq" (heavy-quark) process [13] in revision 3180 of the POWHEGbox [14] generator, which simulates top-quark production to next-to-leading (NLO) accuracy in the strong coupling constant. We use it with the PDF4LHC15_nlo_30 PDF set [15], and shower events with Pythia 8.223 [16,17], tune 4C [18]. Our final results will be based on events at the hadron level, without an underlying event. The number of events that we can expect for an integrated luminosity $\mathcal{L}_{A A}$ of $A A$ collisions is $n(f) \simeq \mathcal{L}_{A A} \sigma_{p p}^{(t \bar{t})} A^{2} c(f)$, where $\sigma_{p p}^{(t \bar{t})}$ is the $p p$ cross section for $t \bar{t}$ production and $A$ is the atomic mass of the ions being collided (see also Ref. [19]). The $c(f)$ factor accounts for the centrality range $f$. We will concentrate on $f=0 \%-10 \%$, and so use $c(0-10 \%) \simeq$ $0.42[20]$.

To keep the analysis and simulation relatively simple, we choose not to embed events in a heavy-ion medium. Instead, we introduce a single factor to mimic the combination of all sources of fluctuations: those from the embedding and medium-subtraction procedure, from finite detector resolution, and also from jet quenching dynamics. Specifically, we rescale the momentum of each particle $i$ by a factor $\left(1+r \sigma_{p_{t}} / \sqrt{p_{t, i}+1 \mathrm{GeV}}\right)$, where $r$ is a Gaussiandistributed random number (different for each particle) with a standard deviation of $1 ; \sigma_{p_{t}}$ is taken to be $1.5 \mathrm{GeV}^{1 / 2}$. This leads to an effective relative jet energy resolution of about $1.5 \mathrm{GeV}^{1 / 2} / \sqrt{p_{t}}$ for high- $p_{t}$ jets, or about $15 \%$ for $p_{t}=100 \mathrm{GeV}$, consistent with Ref. [21].

To simulate baseline full quenching in $0 \%-10 \%$ central $\mathrm{PbPb}$ systems, we apply a constant energy loss rescaling factor $\mathcal{Q}_{0}=0.85$ to all particle momenta, which is consistent with observations in $\gamma / Z+$ jets measurements performed by the ATLAS and CMS Collaborations $[22,23]$. Recall that the fluctuations associated with quenching are included in our single global fluctuation factor. A more sophisticated analysis would be possible, but is perhaps best carried out in the context of a full experimental study.

To account for dependence of the quenching on the time $\tau_{\text {tot }}$ at which the $W$ decay products start to interact with the medium, all particles from the $W$ decay are scaled by a factor $\mathcal{Q}\left(\tau_{\text {tot }}\right)$ rather than $\mathcal{Q}_{0}$. We will return to the exact form of $\mathcal{Q}\left(\tau_{\text {tot }}\right)$ below.

To tag potential $t \bar{t}$ events, we require the presence of a muon, two $b$-tagged jets, and at least two non- $b$-tagged jets. The muon should have $p_{t}>25 \mathrm{GeV}$ and rapidity $|y|<2.5$. Jets are obtained using the anti- $k_{t}$ jet algorithm [24] with radius $R=0.3$ and subsequent partial declustering $[25,26]$ with the $k_{t}$ algorithm [27,28], all performed within FastJet v3.2.1 [29]. A selection requirement of $p_{t}>30 \mathrm{GeV}$ and $|y|<2.5$ is applied to the anti- $k_{t}$ jets. We assume a $b$-tagging efficiency of $\epsilon_{b}=70 \%$ per $b$, as obtained in $\mathrm{pPb}$ events in Ref. [12] and anticipating the expected improvements in $b$ tagging in high-multiplicity environments from HL-LHC detector upgrades [30,31]. We also assume that fake $b$ tags do not introduce any substantial background. 
Our $W$ and top-quark reconstruction procedure is inspired by the pseudo-top definition of Refs. [32,33], adapted to be more resilient to the presence of additional jets from initial-state radiation (ISR) and at the same time robust with respect to effects of quenching on the energy scales of $W$ and top candidates. The use of $R=0.3$ anti- $k_{t}$ clustering and then $k_{t}$ declustering to obtain the input jets helps ensure adequate performance across a broad range of top-quark transverse boosts (similar in spirit though different in its details to Ref. [34]). The full procedure is detailed in the Supplemental Material [8].

For each event that satisfies the reconstruction requirements, we consider two observables: $m_{W}^{\text {reco }}$, the mass of the reconstructed hadronic $W$-boson candidate and $p_{t, \text { top }}^{\text {reco }}$, the $p_{t}$ of the corresponding top candidate. The former will provide our measure of quenching (and was once before studied for this purpose [35]). The latter can be translated to an average $\tau_{\text {tot }}$ and for $200 \mathrm{GeV} \lesssim p_{t, \text { top }}^{\text {reco }} \lesssim 1 \mathrm{TeV}$ the relation reads (see Fig. 6 in the Supplemental Material [8])

$$
\left\langle\tau_{\text {tot }}\right\rangle\left(p_{t, \text { top }}^{\text {reco }}\right) \simeq\left(0.37+0.0022 p_{t, \text { top }}^{\text {reco }} / \mathrm{GeV}\right) \mathrm{fm} / c .
$$

The distribution of $\tau_{\text {tot }}$ values is given in Fig. 1 for the LHC $\sqrt{s_{N N}}=5.5 \mathrm{TeV}$, inclusively over $p_{t, \mathrm{top}}^{\mathrm{reco}}$, and for a futurecircular collider (FCC) with $\sqrt{s_{N N}}=39 \mathrm{TeV}$, considering events with $p_{t, \text { top }}^{\text {reco }}>400 \mathrm{GeV}$. Note the long tails in both cases, which will contribute sensitivity to times substantially beyond $\left\langle\tau_{\text {tot }}\right\rangle$.

Figure 2 shows the distribution of $m_{W}^{\text {reco }}$, again for the LHC and FCC, with a $p_{t, \text { top }}^{\text {reco }}$ cut in the latter case. Results are shown with baseline full quenching for all particles and without quenching (the latter being equivalent to $p p$ events embedded in heavy-ion events to account for the effect of the underlying event). One sees clear $W$-mass peaks, superposed on a continuum associated with events where the $W$ decay jets have not been correctly identified. The continuum is significantly reduced at high $p_{t, \text { top }}^{\text {reco }}$ The $W$ peaks in the quenched case are shifted to the left, and the extent of the shift provides an experimental measure of the

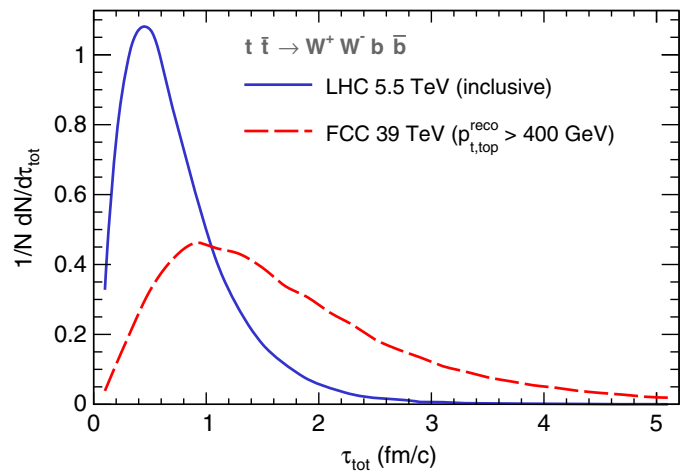

FIG. 1. Distribution of $\tau_{\text {tot }}$ for events that pass all reconstruction cuts and have a top-quark candidate (independently of the reconstructed top-quark and $W$-boson masses). quenching. The peaks are also lower in the quenched case, reflecting the smaller fractions of events that pass the reconstruction (and, for FCC, $p_{t, \text { top }}^{\text {reco }}$ ) cuts.

To estimate the sensitivity of top-quark measurements to the time dependence of quenching in the medium, we consider a toy model in which the quenching is proportional to the time between the moment when the $W$ decay products decohere, $\tau_{\text {tot }}$, and a moment when the medium quenching effect stops being active, $\tau_{m}$. This gives a $\tau_{\text {tot }}$-dependent quenching factor $\mathcal{Q}\left(\tau_{\text {tot }}\right)$ for the $W$ decay products of

$$
\mathcal{Q}\left(\tau_{\text {tot }}\right)=1+\left(\mathcal{Q}_{0}-1\right) \frac{\tau_{m}-\tau_{\text {tot }}}{\tau_{m}} \Theta\left(\tau_{m}-\tau_{\text {tot }}\right) .
$$

Recall that all other hadronic particles undergo quenching with the factor $\mathcal{Q}_{0}$.

For each choice of $\tau_{m}$ we obtain a $m_{W}^{\text {reco }}$ histogram as in Fig. 2. We carry out a binned likelihood fit for the histogram and the background of incorrectly reconstructed $W$ 's using the functional form

$$
N(m)=a \exp \left(-\frac{\left(m-m_{W}^{\mathrm{fit}}\right)^{2}}{2 \sigma^{2}}\right)+b+c m
$$

which yields good fits. The free parameters $a, b, c, \sigma$, and $m_{W}^{\text {fit }}$ are constrained to sensible ranges so as to increase the stability of the fit in low statistics samples.

Figure 3 shows the results for $m_{W}^{\text {fit }}$. They are plotted as bands for different $\tau_{m}$ values, as a function of the $\mathrm{PbPb}$ integrated luminosity, $\mathcal{L}_{\mathrm{PbPb}}$. The width of each band represents the standard deviation of $m_{W}^{\text {fit }}$ values that we obtain when we carry out fits for a large number of replica pseudoexperiments. Two of the bands are independent of the $\mathrm{PbPb}$ luminosity: the top, unquenched band, corresponds to the result that would be obtained by embedding $2 \mathrm{fb}^{-1}$ of $p p$ (unquenched) data into minimum-bias $\mathrm{PbPb}$ events. The bottom band is obtained by a similar procedure,

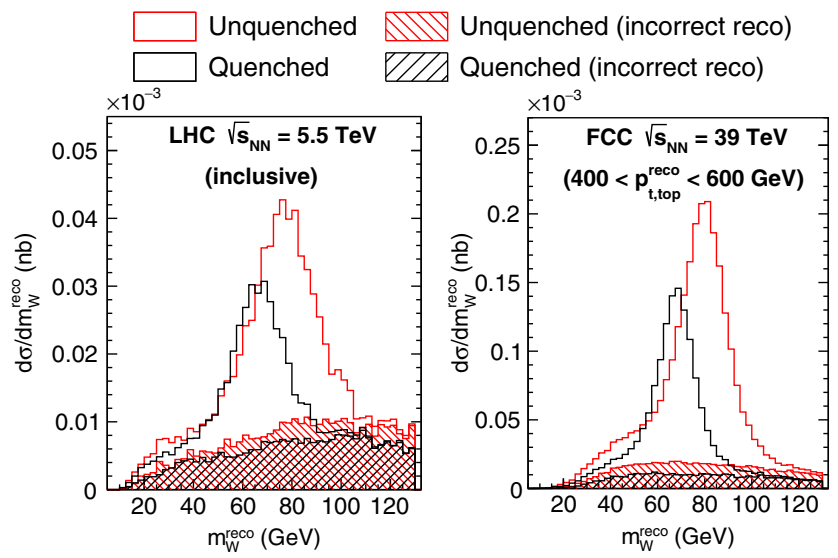

FIG. 2. Differential fiducial proton-proton $t \bar{t}$ reconstruction cross section as a function of $m_{W}^{\text {reco }}$ at the LHC and FCC. 


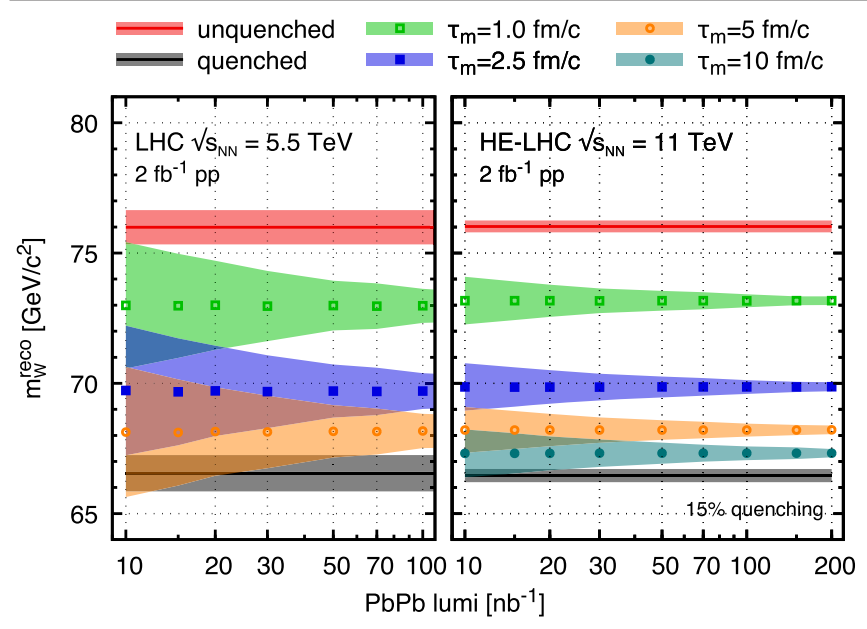

FIG. 3. The average (points) and standard deviation (width of band) for $m_{W}^{\text {reco }}$ across many pseudoexperiments, as a function of luminosity for an inclusive sample of $t \bar{t}$ events, as a function of the integrated $\mathrm{PbPb}$ luminosity at the LHC (left) and the HE-LHC (right).

but with the $p p$ jets' particles simply scaled down by the quenching factor $\mathcal{Q}_{0}$, i.e., by the quenching factor that would be expected if the $W$ decay products were present and started interacting from time 0 . In a real experiment, the corresponding scaling factor could be obtained by measuring quenching in another quark-jet dominated process (e.g., with $\gamma+$ jet or $Z+$ jet balance), as a function of the jet $p_{t}$.

For short values of the effective medium lifetime, $\tau_{m}$, the $m_{W}^{\mathrm{fit}}$ result is close to the unquenched result. This reflects the fact that the $W$ decay products start interacting only towards the end of the medium lifetime. For larger values of $\tau_{m}$ they instead still see most of the medium duration, and most of the quenching. A very short-lived medium, $\tau_{m}=1 \mathrm{fm} / c$, could be distinguished from the full quenching baseline at the LHC with its currently approved $\mathcal{L}_{\mathrm{PbPb}}=10 \mathrm{nb}^{-1}$. However, to distinguish larger values of $\tau_{m}$ would require either higher luminosities or higher energies. This is illustrated in the right-hand plot of Fig. 3 for a future $\mathrm{HE}-\mathrm{LHC}\left(\sqrt{s_{N N}}=11 \mathrm{TeV}\right)$, where the $t \bar{t}$ cross section is 6 times larger.

At higher-energies it becomes advantageous to explore the $p_{t, \text { top }}^{\text {reco }}$ dependence of $m_{W}^{\text {fit }}$, illustrated in Fig. 4 for the HE-LHC and the FCC $\left(\sqrt{s_{N N}}=39 \mathrm{TeV}\right)$. For each bin of $p_{t, \text { top }}^{\text {reco }}$, the upper axis shows the corresponding average $\tau_{\text {tot }}$. For a given band of $\tau_{m}$, when $p_{t, \text { top }}^{\text {reco }}$ is large enough so that $\left\langle\tau_{\text {tot }}\right\rangle \gtrsim \tau_{m}$, the band merges with the unquenched expectation. Thus the shape of the $p_{t, \text { top }}^{\text {reco }}$ dependence gives powerful information on the medium time structure. (The unquenched and baseline-quenched bands also have a $p_{t, \text { top }}^{\text {reco }}$ dependence, induced by the underlying jet and muon $p_{t}$ cuts, as well as different amounts of final-state radiation outside the $R=0.3$ jet as a function of $p_{t, \text { top }}^{\text {reco }}$.)

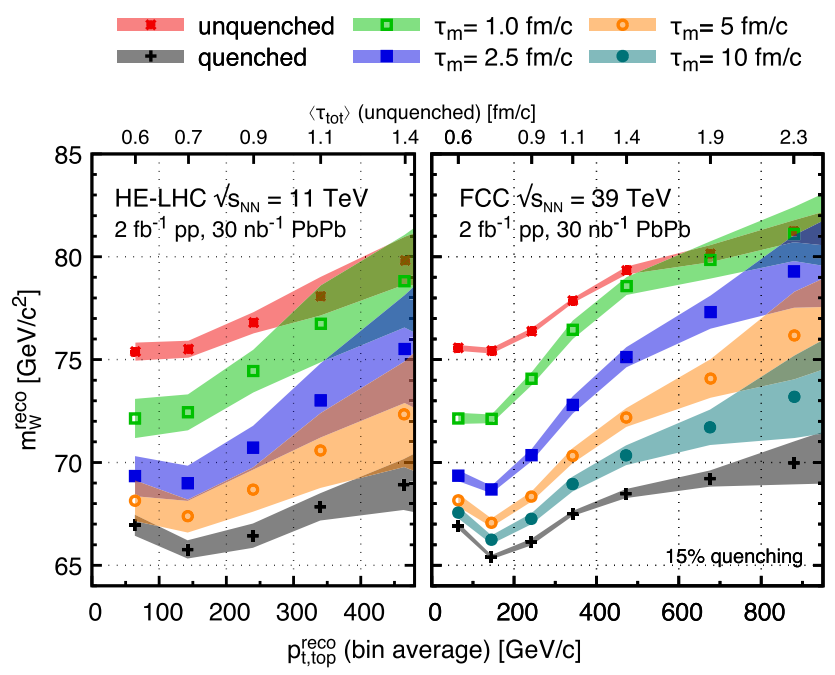

FIG. 4. Dependence of the reconstructed $W$ mass on the reconstructed top $p_{t}$ for HE-LHC (left) and FCC (right) collisions. The quenched result corresponds to baseline full modification of the $p p$ results, which would in practice be obtained using knowledge of quenching from other measurements.

Figure 5 shows our estimate of the maximum $\tau_{m}$ that can be distinguished at 2 standard deviations from the baseline full quenched result, for different colliders $[36,37]$ as a function of $\mathcal{L}_{\mathrm{PbPb}}$. The number of standard deviations takes into account the statistical uncertainty of $m_{W}^{\mathrm{fit}}$, for both the actual heavy-ion data and a reference sample, as well as an additional $1 \%$ systematic uncertainty (see Supplemental Material [8] and Refs. [22,38]). The reference sample is obtained using the same procedure as for the bottom bands in Figs. 3 and 4, i.e., using $2 \mathrm{fb}^{-1}$ of $p p$ events with a rescaling of particle momenta by a factor $\mathcal{Q}_{0}$ and inclusion of underlying-event fluctuations.

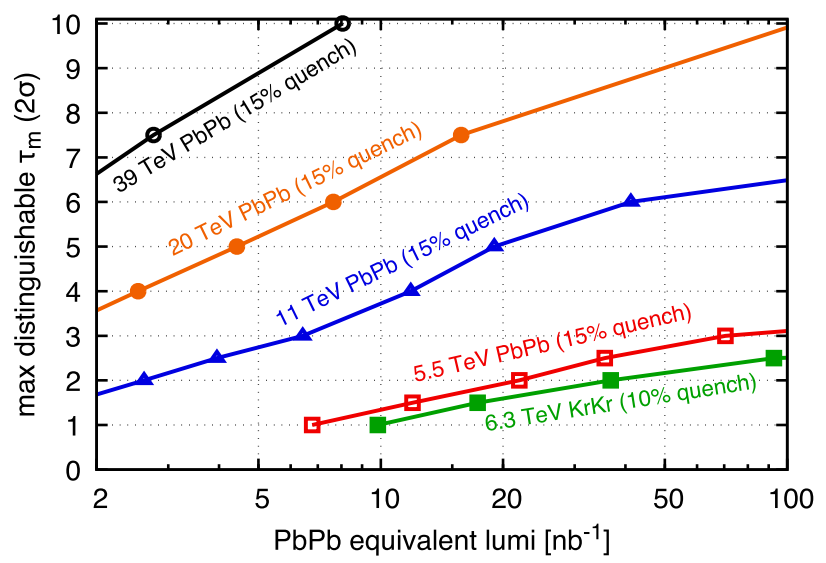

FIG. 5. The maximum medium quenching end time $\tau_{m}$ that can be distinguished from full quenching with 2 standard deviations, as a function of luminosity for different collider energies [36,37] and species. For the $\mathrm{KrKr}$ points, the $\mathcal{L}_{\mathrm{KrKr}}$ value that is used is equal to $\mathcal{L}_{\mathrm{PbPb}}\left(A_{\mathrm{Pb}} / A_{\mathrm{Kr}}\right)^{2}$, i.e., maintaining an equal number of nucleon-nucleon collisions. 
For each collider luminosity and energy the results are obtained by choosing a $p_{t, \text { top }}^{\text {reco }}$ cut so as to maximize the significance. We have verified that if we increase the fluctuations, $\sigma_{p_{t}}$, the required luminosity scales as $\sigma_{p_{t}}^{2}$, in line with expectations.

Lighter ions such as $\mathrm{Kr}$ are potentially promising, despite their smaller quenching effects [39], because of the potential for order-of-magnitude higher effective integrated nucleon-nucleon luminosities [40,41]. They are discussed further in the Supplemental Material [8].

To conclude, in this work we have shown that the study of top quarks and their decays has a unique potential to resolve the time dimension in jet-quenching studies of the QGP. To benefit from this potential requires a sufficiently large sample of top quarks, in particular to enhance event rates on the high- $p_{t}$ tail, which gives the sensitivity to the longer timescales. At the LHC, with currently planned luminosity, such a program could begin. With higher energy colliders or a significantly increased luminosity at the LHC (whether from longer running or lighter ion species), there would be substantial prospects for using jet quenching to study the evolution of the QGP over the first few $\mathrm{fm} / c$. Overall, our results provide a strong motivation for a program of experimental studies of top-quark production in heavy-ion collisions.

We are grateful to John Jowett for bringing to our attention the potential for higher nucleon-nucleon luminosities with ions lighter than $\mathrm{Pb}$ and to Detlef Küchler for information on possible ion species in the LHC. We would also like to thank Andrea Giammanco for exchanges regarding pseudotop definitions, Phil Harris for discussions concerning the use of $W+$ jet events, and Roberto Franceschini for a comment on the manuscript. This work was supported in part by the Fundação para a Ciência e Tecnologia (Portugal) under contracts CERN/FIS-NUC/ 0049/2015 (L. A. and J. G. M.), Investigador FCTDevelopment Grant IF/00563/2012 (JGM) and SFRH/ BPD/103196/2014 (L. A.); and by European Research Council Grant No. HotLHC ERC-2011-StG-279579, by Ministerio de Ciencia e Innovacion of Spain under Project No. FPA2014-58293-C2-1-P and Maria de Maetzu Unit of Excellence MDM-2016-0692, by Xunta de Galicia AGRUP2015/11 (CAS).

* On leave from CNRS, UMR 7589, LPTHE, F-75005, Paris, France.

[1] N. Armesto and E. Scomparin, Eur. Phys. J. Plus 131, 52 (2016).

[2] C. Gale, S. Jeon, and B. Schenke, Int. J. Mod. Phys. A 28, 1340011 (2013).

[3] C. Loizides, Nucl. Phys. A956, 200 (2016).

[4] C. A. Salgado and J. P. Wessels, Annu. Rev. Nucl. Part. Sci. 66, 449 (2016).
[5] Y. Mehtar-Tani, J. G. Milhano, and K. Tywoniuk, Int. J. Mod. Phys. A 28, 1340013 (2013).

[6] In light of Ref. [7], similar measurements of the time structure of the QGP could be accessible with $W+$ jet events. However, we will focus here on the $t \bar{t}$ avenue which we consider more promising (cf. the Supplemental Material [8]).

[7] A. M. Sirunyan et al. (CMS Collaboration), Phys. Rev. Lett. 119, 111802 (2017).

[8] See Supplemental Material at http://link.aps.org/ supplemental/10.1103/PhysRevLett.120.232301, for a discussion of the advantages of using top + anti-top events in comparison with $W+$ jet events, a detailed description of the adopted top reconstruction procedure, a breakdown of the contributions to the total delay time, the justification of our assumed control of the jet energy scale, and a brief discussion of opportunities afforded by collision of lighter ion species.

[9] J. Casalderrey-Solana, Y. Mehtar-Tani, C. A. Salgado, and K. Tywoniuk, Phys. Lett. B 725, 357 (2013).

[10] K. M. Burke et al. (JET Collaboration), Phys. Rev. C 90, 014909 (2014).

[11] C. Andres, N. Armesto, M. Luzum, C. A. Salgado, and P. Zurita, Eur. Phys. J. C 76, 475 (2016).

[12] A. M. Sirunyan et al. (CMS Collaboration), Phys. Rev. Lett. 119, 242001 (2017).

[13] S. Frixione, P. Nason, and G. Ridolfi, J. High Energy Phys. 09 (2007) 126.

[14] S. Alioli, P. Nason, C. Oleari, and E. Re, J. High Energy Phys. 06 (2010) 043.

[15] J. Butterworth et al., J. Phys. G 43, 023001 (2016).

[16] T. Sjostrand, S. Mrenna, and P. Z. Skands, J. High Energy Phys. 05 (2006) 026.

[17] T. Sjostrand, S. Mrenna, and P. Z. Skands, Comput. Phys. Commun. 178, 852 (2008).

[18] R. Corke and T. Sjostrand, J. High Energy Phys. 03 (2011) 032.

[19] D. d'Enterria, K. Krajczár, and H. Paukkunen, Phys. Lett. B 746, 64 (2015).

[20] C. Loizides, J. Kamin, and D. d'Enterria, arXiv:1710.07098 [Phys. Rev. C (to be published)].

[21] A. M. Sirunyan et al. (CMS Collaboration), Phys. Rev. Lett. 119, 082301 (2017).

[22] ATLAS Collaboration, Study of photon-jet momentum correlations in $\mathrm{Pb}+\mathrm{Pb}$ and $p p$ collisions at $\sqrt{s_{\mathrm{NN}}}=$ $5.02 \mathrm{TeV}$ with ATLAS, Technical Report No. ATLASCONF-2016-110 CERN, Geneva, 2016.

[23] CMS Collaboration, Study of $Z+$ jet correlations in $\mathrm{PbPb}$ and pp collisions at $\sqrt{s_{N N}}=5.02 \mathrm{TeV}$, Technical Report No. CMS-PAS-HIN-15-013 CERN, Geneva, 2016.

[24] M. Cacciari, G. P. Salam, and G. Soyez, J. High Energy Phys. 04 (2008) 063.

[25] M. H. Seymour, Z. Phys. C 62, 127 (1994).

[26] J. M. Butterworth, B. E. Cox, and J. R. Forshaw, Phys. Rev. D 65, 096014 (2002).

[27] S. Catani, Y. L. Dokshitzer, M. H. Seymour, and B. R. Webber, Nucl. Phys. B406, 187 (1993).

[28] S. D. Ellis and D. E. Soper, Phys. Rev. D 48, 3160 (1993).

[29] M. Cacciari, G. P. Salam, and G. Soyez, Eur. Phys. J. C 72, 1896 (2012). 
[30] ATLAS Collaboration, ATLAS Phase-II Upgrade Scoping Document, Technical Report No. CERN-LHCC-2015-020. LHCC-G-166 CERN, Geneva, 2015.

[31] CMS Collaboration, Technical Proposal for the Phase-II Upgrade of the CMS Detector, Technical Report No. CERN-LHCC-2015-010. LHCC-P-008. CMS-TDR15-02 CERN, Geneva, 2015.

[32] G. Aad et al. (ATLAS Collaboration), J. High Energy Phys. 06 (2015) 100.

[33] CMS Collaboration, Object definitions for top quark analyses at the particle level, Technical Report No. CERN-CMSNOTE-2017-004 CERN, Geneva, 2017.

[34] M. Gouzevitch, A. Oliveira, J. Rojo, R. Rosenfeld, G. P. Salam, and V. Sanz, J. High Energy Phys. 07 (2013) 148.

[35] L. Bhattacharya, K. Ghosh, and K. Huitu, arXiv:1210.0116.
[36] A. Dainese et al., CERN Yellow Rep. Monogr. 3, 635 (2017).

[37] N.-B. Chang et al., Sci. China Phys. Mech. Astron. 59, 621001 (2016).

[38] CMS Collaboration, Projected Heavy Ion Physics Performance at the High Luminosity LHC Era with the CMS Detector, Technical Report No. CMS-PAS-FTR-17-002 CERN, Geneva, 2017.

[39] B. I. Abelev et al. (STAR Collaboration), Phys. Rev. C 81, 054907 (2010).

[40] J. M. Jowett, Colliding heavy ions in the LHC, Proceedings of 9th International Particle Accelerator Conference (IPAC 2018), Vancouver (2018), http://ipac2018.vrws.de/papers/ tuxgbd2.pdf.

[41] R. Bruce, J. M. Jowett, S. Gilardoni, A. Drees, W. Fischer, S. Tepikian, and S. R. Klein, Phys. Rev. Lett. 99, 144801 (2007). 